\title{
AUTOMORPHISM GROUPS ON COMPACT RIEMANN SURFACES
}

\author{
BY \\ W. T. KILEY( $\left.{ }^{1}\right)$
}

\begin{abstract}
For $g \geqq 2$, let $N(g)$ be the order of the largest automorphism group on a Riemann surface of genus $g$. In this paper, lower bounds for $N(g)$ for various sequences of $g$ 's are obtained. Sequences of appropriate groups are constructed. Each of these groups is then realized as a group of cover transformations of a surface covering the Riemann sphere. The genus of the resulting surface is then found by using the Riemann-Hurwitz formula and the automorphism group of the surface contains the given group. Each lower bound which is found is also shown to be sharp. That is, there are infinitely many $g$ 's in the sequence to which the bound applies for which $N(g)$ does not exceed the bound.
\end{abstract}

1. Introduction. Let $W$ be a Riemann surface and let $A(W)$ be the group of its automorphisms (conformal self-maps). Assume $W$ is a compact surface of genus $g$. If $g<2, A(W)$ is infinite. Henceforth, we assume $g \geqq 2$. For such $g$, the order of $A(W)$ depends on the conformal type of $W$ as well as on its genus. Hence we define $N(g)$ to be the order of the largest group on a surface of genus $g$. It is the determination of this $N(g)$ for various $g$ 's which shall be our principal concern.

Hurwitz has shown that $N(g) \leqq 84(g-1)$ for all $g$ [2]. Macbeath proved that this bound is sharp, i.e., for infinitely many $g$ 's the bound is attained [6]. He also showed that for infinitely many $g$ 's, $N(g)<84(g-1)$ and hence this upper bound does not solve the problem [5].

Accola [1] and Maclachlan [7] proved independently that $N(g) \geqq 8(g+1)$ for all $g$. They have also shown that $N(g) \geqq 8(g+3)$ for the arithmetic sequence of $g$ 's which are divisible by 3 . In addition, they have establishęd that both of these bounds are sharp. These results shall serve as the prototype for the work of this paper.

In $\$ 3$ we show that for any positive integer $l, N(g) \geqq 8\left(g-1+2^{l}\right)$ if $g \equiv\left(1-2^{l}\right)$ $\left(\bmod l 2^{l-1}\right)$; for any positive integers $l$ and $h$, where $h$ divides $l, N(g) \geqq 8\left(g-1+2^{l}\right)$

Received by the editors November 3, 1969 and, in revised form, December 15, 1969.

AMS Subject Classifications. Primary 3080; Secondary 2025.

Key Words and Phrases. Automorphism group, compact Riemann surface, covering surfaces, fundamental group, Riemann-Hurwitz formula, semidirect product group, permutation group.

( ${ }^{1}$ ) A major portion of this paper was first produced as part of my thesis at Brown University under the guidance of R. D. M. Accola. I wish to reiterate my appreciation for his contribution to that work. 
if $g \equiv\left(1-2^{l}\right)\left(\bmod 2^{l-2}(l+h)\right)$; and for any positive integer $n, N(g) \geqq 8\left(g-1+2 n^{2}\right)$ if $g \equiv\left(1-2 n^{2}\right)\left(\bmod n^{3}\right)$. In $\S 4$ each of these lower bounds is shown to be sharp. The results of Accola and Machlachlan are special cases of these results.

2. Covering surfaces. Assume $(W, f)$ is an unlimited $n$-sheeted covering of $W_{0}$. The following theorem will be essential to much of this paper.

2.1 Riemann-Hurwitz formula [2]. If $W$ and $W_{0}$ are compact surfaces of genera $g$ and $g_{0}$, respectively, and $r$ is the total ramification of the covering, then

$$
2(g-1)=n 2\left(g_{0}-1\right)+r .
$$

Let $E_{0}$ be the points of $W_{0}$ above which the covering is branched and let $E=f^{-1}\left(E_{0}\right)$. Let $W_{0}^{\prime}=W_{0}-E_{0}, W^{\prime}=W-E$, and $f^{\prime}$ be the restriction of $f$ to $W^{\prime}$. Then $\left(W^{\prime}, f^{\prime}\right)$ is a smooth unlimited $n$-sheeted covering of $W_{0}^{\prime}$. The group $G$ of cover transformations of the covering $W$ of $W_{0}$ and the group $G^{\prime}$ of cover transformations of the covering $W^{\prime}$ of $W_{0}^{\prime}$ are naturally isomorphic.

Let $F_{0}$ be the fundamental group of $W_{0}^{\prime}$ based at a point $p_{0}$. Let $\left\{p_{1}, \ldots, p_{n}\right\}$ be the set of points above $p_{0}$. If $\gamma_{0} \in F_{0}$, let $\gamma_{i}$ be the continuation along $\gamma_{0}$ from $p_{i}$ for $i=1, \ldots, n$. Let $\mu\left(\gamma_{0}\right)$ map $p_{i}$ into the end point of $\gamma_{i}$. The image of $\mu$ is a transitive subgroup of $S_{n}$, the symmetric group on $n$ objects. The mapping $\mu$ is a homomorphism of $F_{0}$ into $S_{n}$ (we will multiply permutations from left to right). We shall refer to $\mu$ as the induced representation of the covering.

The branching of the covering $W$ of $W_{0}$ is related to $\mu$ in the following fashion. Take $q_{0} \in E_{0}$ and a $\gamma_{0} \in F_{0}$ which "circles" $q_{0}$. The multiplicities at the branch points above $q_{0}$ will then coincide with the lengths of the cycles of $\mu\left(\gamma_{0}\right)$. Note that if $\mu\left(F_{0}\right)$ is to be a regular subgroup of $S_{n}$, then the multiplicities above a given point must be equal. In this case, if the branching occurs above $q_{i}$ with multiplicity $m_{i}$ for $i=1, \ldots, s$, then the Riemann-Hurwitz formula reduces to:

$$
2(g-1)=n\left[2\left(g_{0}-1\right)+\sum_{i=1}^{s}\left(1-\frac{1}{m_{i}}\right)\right] .
$$

We will relate $G \approx G^{\prime}$ and $\mu$ by means of the following theorem.

\subsection{THEOREM [10]. $\mu\left(F_{0}\right)$ is a regular subgroup of $S_{n}$ if and only if $G^{\prime} \approx \mu\left(F_{0}\right)$.}

A covering which satisfies these conditions will be called normal. All the coverings with which we will be concerned will be normal.

The above procedure can be reversed. Let $W_{0}^{\prime}=W_{0}-E_{0}$ where $E_{0}$ is a discrete subset of $W_{0}$. Let $F_{0}$ be the fundamental group of $W_{0}^{\prime}$. Assume $\mu$ is a homomorphism of $F_{0}$ into $S_{n}$ whose image is transitive. A smooth unlimited $n$-sheeted covering $W^{\prime}$ of $W_{0}^{\prime}$ which induces $\mu$ can then be constructed [8]. Finally, the covering $W^{\prime}$ can be extended in a unique fashion to give an unlimited $n$-sheeted covering $W$ of $W_{0}$ which is branched at most above the points of $E_{0}$. 
3. New lower bounds. Our method will be to realize certain abstract groups as a group of cover transformations of a surface covering the Riemann sphere. Let $W_{0}$ be the Riemann sphere and let $E_{0}=\left\{q_{1}, q_{2}, q_{3}\right\}$ be a set of three points on it. Let $W_{0}^{\prime}=W_{0}-E_{0}$. Choose $\gamma_{1}, \gamma_{2}$, and $\gamma_{3}$ so that each $\gamma_{i}$ circles $q_{i}$ and so that the fundamental group $F_{0}$ of $W_{0}^{\prime}$ has generating set $\left\{\gamma_{1}, \gamma_{2}, \gamma_{3}\right\}$ with defining relation $\gamma_{1} \gamma_{2} \gamma_{3}=1$.

Now let $H$ be a group with generating set $\{a, b\}$ such that the orders of $a, b$ and $a b$ are $\alpha, \beta$, and $\lambda$, respectively. Such a group will be called an $(\alpha, \beta, \lambda)$ group. $H$ is isomorphic to a regular, transitive subgroup of $S_{n}$, where $n$ is the order of $H$.

Define $\mu\left(\gamma_{1}\right)=a, \mu\left(\gamma_{2}\right)=b$, and $\mu\left(\gamma_{3}\right)=(a b)^{-1}$. Extend $\mu$ to a homomorphism of $F_{0}$ onto $H$. There is then an unlimited $n$-sheeted normal covering $(W, f)$ of $W_{0}$ which induces $\mu$. The group of cover transformations of this covering is isomorphic to $H$. $W$ can be given a conformal structure so that $f$ is analytic and hence each cover transformation is an automorphism.

Let $m, t$, and $s$ be positive integers. Assume $H$ is a $(2,4, m t)$ group and $n=2 m t s$. The genus $g$ of $W$ by (1) equals $1-s+m(t s / 4)$. Therefore $n=8(g-1+s)$. Hence we have proven:

3.1 LEMMA. If for fixed positive integers $s$ and $t$ and for each positive integer $m$ there is $a(2,4, m t)$ group of order $2 m t s$, then $N(g) \geqq 8(g-1+s)$ if

$$
g \equiv(1-s)(\bmod (s t / 4))
$$

The following lemma will be used to construct groups to which we will apply the previous lemma.

3.2 Lemma. Let $l$ and $k$ be positive integers. Assume $\phi$ is a group automorphism of $\left(Z_{2}\right)^{l}$ whose order divides $k$. Assume there is an $x$ such that $\left\{x, \phi(x), \ldots, \phi^{l-1}(x)\right\}$ generates $\left(Z_{2}\right)^{l}$. Let $r$ be the order of $x \cdot \phi(x) \cdots \phi^{k-1}(x)$. Define an automorphism $\theta$ of $\left(Z_{2}\right)^{l}$ by $\theta\left(\phi^{j}(x)\right)=\phi^{1-j}(x)$ for $j=0, \ldots, l-1$. If $\theta\left(\phi^{l}(x)\right)=\phi^{1-l}(x)$, then for every $m$ there is $a(2,4, m k r)$ group of order $2^{l+1} m k r$.

Proof. Let $d$ generate $Z_{m k r}$. Form a semidirect product of $\left(Z_{2}\right)^{l}$ by $Z_{m k r}$ by defining $d y d^{-1}=\phi(y)$ for each $y$ in $\left(Z_{2}\right)^{l}$ [9]. This product is generated by the set $\left\{d, x, \phi(x), \ldots, \phi^{l-1}(x)\right\}$ with defining relations: $d^{m k r}=x^{2}=1, d \cdot \phi^{j}(x) \cdot d^{-1}=$ $\phi^{j+1}(x)$ for $j=0,1, \ldots, l-1$ and $x \cdot \phi^{j}(x)=\phi^{j}(x) \cdot x$ for $j=1, \ldots, l-1$. Now extend $\theta$ to this group by defining $\theta(d)=x d^{-1}$. Observe that .

$$
\left(x d^{-1}\right)^{k}=d^{-k}\left(d^{k} x d^{-k}\right)\left(d^{k-1} x d^{1-k}\right) \cdots\left(d x d^{-1}\right)=d^{-k} \cdot x \cdot \phi^{k-1}(x) \cdots \phi(x) .
$$

So $\left(x d^{-1}\right)^{k r}=d^{-k r} \cdot \theta$ is well defined since it preserves the relations. The order of $\theta$ is 2 . Hence we can form a semidirect product $H$ of the above group by $Z_{2}$.

Let $a$ be the generator of $Z_{2}$. Let $b=a d$. Then $\{a, b\}$ generates $H$. The orders of $a, b$, and $a b$ are 2,4 , and $m k r$, respectively. Hence $H$ satisfies the desired conditions. Q.E.D. 
In each of the following two applications, let $\left\{x_{1}, \ldots, x_{l}\right\}$ be a generating set of $\left(Z_{2}\right)^{l}$.

First define $\phi\left(x_{j}\right)=x_{j+1}$ for $j=1, \ldots, l-1$ and $\phi\left(x_{l}\right)=x_{1}$. Then $\phi$ satisfies the hypothesis of 3.2 with $k=l$ and $r=2$. So there are $(2,4,2 \mathrm{~lm})$ groups of order $2^{l+2} \mathrm{ml}$. Hence we can apply 3.1 with $t=2 l$ and $s=2^{l}$ to obtain:

3.3 TheOREM. For any positive integer $l, N(g) \geqq 8\left(g-1+2^{l}\right)$ if

$$
g \equiv\left(1-2^{l}\right)\left(\bmod l 2^{l-1}\right) \text {. }
$$

Now assume $h$ is an integer which divides $l$. Define $\phi\left(x_{j}\right)=x_{j+1}$ for $j=1, \ldots, l-1$ and $\phi\left(x_{l}\right)=x_{1} \cdot x_{1+h} \cdots x_{1+l-h}$. Then $\phi$ satisfies the hypothesis of 3.2 with $k=l+h$ and $r=1$. So there are $(2,4, m(l+h))$ groups of order $2^{l+1} m(l+h)$. Apply 3.1 with $t=l+h$ and $s=2^{l}$ to obtain:

3.4 Theorem. For any positive integers $l$ and $h$ such that $h$ divides $l, N(g) \geqq$ $8\left(g-1+2^{l}\right)$ for $g \equiv\left(1-2^{l}\right)\left(\bmod 2^{l-2}(l+h)\right)$.

The final construction is somewhat more complicated, but it yields new lower bounds.

Let $m$ and $n$ be any positive integers. Let $x$ generate $Z_{n}$. Define automorphisms $\phi$ and $\theta$ of $Z_{n}$ by $\phi(x)=x$ and $\theta(x)=x^{-1}$. Note that $\theta \circ \phi=\phi^{-1} \circ \theta$. Let $D_{n}$ be the dihedral group generated by $\{R, V\}$ with relations $R^{n}=V^{2}=1$ and $V R=R^{-1} V$. Form a semidirect product of $Z_{n}$ by $D_{n}$ by defining $R x R^{-1}=\phi(x)$ and $V x V^{-1}=\theta(x)$.

Now define an automorphism $\tau$ of the product group by $\tau(x)=x, \tau(R)=x^{-1} R^{-1}$, and $\tau(V)=R V$. It is easily checked that $\tau$ preserves the relations and is of order $2 n$. Let $d$ generate $Z_{2 n m}$. Form the semidirect product of the above group by $Z_{2 n m}$.

Finally, define an automorphism $\sigma$ of the new group by $\sigma(d)=V d^{-1}, \sigma(V)=R V$, $\sigma(R)=R^{-1}$, and $\sigma(x)=x R^{2}$. The required relations are preserved by $\sigma$ and $\sigma$ is of order 2 . So form the semidirect product of the above group by $Z_{2}$. This group is a $(2,4,2 n m)$ group of order $8 n^{3} m$. Apply 3.1 with $t=2 n$ and $s=2 n^{2}$ to obtain:

3.5 TheOREM. For any positive integer $n, N(g) \geqq 8\left(g-1+2 n^{2}\right)$ if $g \equiv\left(1-2 n^{2}\right)$ $\left(\bmod n^{3}\right)$.

It should be added that the groups used in this paper were not initially constructed by the author in the manner described herein. Rather they were obtained by lifting automorphism groups on a surface $W_{0}$ to an $n$-sheeted unlimited normal covering $(W, f)$ of $W_{0}$ by a method developed by Macbeath [6] and Accola [1]. An automorphism $\phi_{0}$ posssesses $n$ distinct lifts $\phi$ such that $f \circ \phi=\phi_{0} \circ f$ if and only if $\phi_{0}$ permutes the branch points and leaves invariant the kernel of the induced representation when acting on $F_{0}$. We illustrate the method by constructing the groups of 3.5. Other examples can be found in [3].

Given positive integers $n$ and $m$, let $c=e(\pi i / n m)$ and let $q_{j}=c^{j}$. Let $W_{0}$ be the Riemann sphere and $E_{0}=\left\{q_{1}, \ldots, q_{2 n m}\right\}$. The fundamental group $F_{0}$ of $W_{0}-E_{0}$ has a generating set $\left\{\gamma_{1}, \ldots, \gamma_{2 n m}\right\}$ where each $\gamma_{j}$ circles $q_{j}$. Take the dihedral 
group generated by $\{R, V\}$ where $R^{n}=V^{2}=1$ and $R V=V R^{-1}$. Define a homomorphism $\mu$ by

$$
\begin{aligned}
\mu\left(\gamma_{j}\right) & =V R & & \text { if } j \text { is odd, } \\
& =V & & \text { if } j \text { is even. }
\end{aligned}
$$

Let $D$ be the kernel of $\mu$.

Define automorphisms $\phi_{0}$ and $\theta_{0}$ of $W_{0}$ by $\phi_{0}(z)=c z$ and $\theta_{0}(z)=1 / z$. Unfortunately $\theta_{0}$ does not leave $D$ invariant. Let $\tilde{D}=D \cap \theta_{0}(D)$. The index of $\tilde{D}$ in $D$ is $n$ (assume $n$ is odd). There exists a $2 n^{2}$-sheeted unlimited normal covering $\tilde{W}$ of $W_{0}$ such that the kernel of the induced representation is $\tilde{D}$. Each member of the group generated by $\left\{\phi_{0}, \theta_{0}\right\}$ can be lifted to $\tilde{W}$. The group of these lifts is then a $(2,4,2 \mathrm{~nm})$ group of order $8 m n^{3}$.

4. Sharpness of the bounds. The goal of this section will be to show that each lower bound obtained in the previous section is sharp. The device which will enable us to accomplish this task is the following theorem.

4.1 TheOrem. Fix $s$ and $t$ where 4 divides st and $s \geqq 2$. Then there are infinitely many $g$ such that $N(g) \leqq 8(g-1+s)$ and $g \equiv(1-s)(\bmod (s t / 4))$.

Observe that 4.1 shows that any bound obtained by applying 3.1 with $s \geqq 2$ is sharp. Since every bound under consideration was obtained in this fashion, the proof of 4.1 will suffice for our purposes. The proof will be based on the following compilation of some theorems by Accola [1].

4.2 LeMma. Assume $G$ is an automorphism group of order $n$ on a surface of genus $g$ where $n \geqq 8(g+1)$. If there are integers $p$ and $s$ with $p$ prime such that $g=p s+1$, $p>84 s, n>(12 s)^{3}$, and $((p-1) / 2,(s+2) !(2 s+3))=1$; then $p$ does not divide $n$ and $G$ is $a(2,4, \lambda)$ group.

The above leads us to further consider $(2,4, \lambda)$ groups.

4.3 Lemma. Assume $G$ is a $(2,4, \lambda)$ group of order $n=\mu \lambda$ with $\mu>4$. Then there is a $k$ dividing $\lambda$ such that $3 \leqq k \leqq \mu$. In addition, $k=3$ only if $\mu=8$. Furthermore, if $\lambda>\mu^{2}$ and 4 divides $\mu$, then $k \leqq \mu / 2$.

Proof. Let $G$ be generated by $a$ and $b$ with orders 2 and 4, respectively. Let $c=a b$ and $d=b a$. Let $k$ be the index of $\langle c\rangle \cap\langle d\rangle$ in $\langle c\rangle$ and let $m$ be the index of $\langle c, d\rangle$ in $G$. Both $\langle c\rangle \cap\langle d\rangle$ and $\langle c, d\rangle$ are normal in $G=\langle a, c\rangle$. Thus $G$ equals the product $\langle c, d\rangle \cdot\langle a\rangle$. Hence $m$ will be 1 or 2 depending on whether or not $a$ is in $\langle c, d\rangle$. Since $\mu\rangle 2$, the order of $\langle c, d\rangle$ is larger than $\lambda$. So neither $b^{2}$ nor $a$ is in $\langle c\rangle$ since $\langle c, d\rangle=\left\langle c, b^{2}\right\rangle$ and $\langle c, d\rangle \subset\langle c, a\rangle$.

Now consider the possible values of $k$. If $k=1$, then $\langle c\rangle \cap\langle d\rangle=\langle c\rangle=\langle c, d\rangle$. So $\mu / m$ will be 1 , which is impossible. If $k=2$, then $\langle c, d\rangle /\langle c\rangle \cap\langle d\rangle$ is a $(2,2,2)$ group and so has order 4. This implies $\mu / m=2$, which is also impossible. If $k=3$, then $G /\langle c\rangle \cap\langle d\rangle$ is a $(2,4,3)$ group, that is $S_{4}$. Thus $3 \mu$ must be 24 . 
From the facts that $c^{j} \notin\langle c\rangle \cap\langle d\rangle$ for $j=1, \ldots, k-1$ and $d^{i} \neq 1$ for $i=1, \ldots$, $\lambda-1$, it follows that the elements $c^{j} d^{i}$ for $j=1, \ldots, k$ and $i=1, \ldots, \lambda$ form $k \lambda$ distinct elements of $\langle c, d\rangle$. Hence $k \lambda \leqq n / m$, i.e. $k \leqq \mu / m$. Now impose the assumptions $m=1$ and 4 divides $\mu$. Clearly $\langle c\rangle \cap\langle d\rangle$ is central in $\langle c, d\rangle=G$. So the transfer homomorphism of $G$ into $\langle c\rangle \cap\langle d\rangle$ reduces to $g_{-} \rightarrow g^{k \mu}$ [11]. This homomorphism must map $a$ and $b$, and therefore $c$, into the identity. So $\lambda$ divides $k \mu$ and hence $\lambda \leqq k \mu \leqq \mu^{2}$. Q.E.D.

We are now prepared to prove 4.1. First choose a $c$ which is divisible by every $k \leqq 4 s$ and by $t$ and such that $c / 2$ is divisible by every prime factor of $(s+2) !(2 s+3)$. Next consider those primes $p$ such that $p \equiv-1(\bmod c)$ and $p \geqq 6^{3} s^{2}$. By Dirichlet's theorem infinitely many such $p$ exist [4]. Let $g=p s+1$ where $p$ is one of these primes. Then $g \equiv(1-s)(\bmod s c) \equiv(1-s)(\bmod (s t / 4))$. So each such $g$ belongs to the sequence with which 4.1 is concerned.

Assume on a surface of the given genus $g$ there is a group $G$ with order $n \geqq 8(g+1)$. Since $(p-1) / 2 \equiv-1(\bmod (c / 2))$, we have $((p-1) / 2,(s+2) !(2 s+3))=1$. Also $n \geqq 8(g+1)=8(p s+2)>(12 s)^{3}$. So by $4.2, p$ does not divide $n$ and $G$ is a $(2,4, \lambda)$ group of order $n=\mu \lambda$. Formula (1) for the covering $W \rightarrow W / G$ is $n=8(g-1+\mu / 2)$. Our problem then reduces to showing $\mu \leqq 2 s$. Expressing $g$ in terms of $p$ gives:

$$
\mu(\lambda-4)=8 p s \text {. }
$$

Since $p$ does not divide $\mu, \mu$ divides $8 s$. Consequently, $\mu$ equals $8 s, 4 s$, or $8 s / 3$ or $\mu \leqq 2 s$. Assume $\mu=8 s, 4 s$, or $8 s / 3$. Then $\mu>4$ and 4 divides $\mu$. Also $\lambda>8 p s / \mu$ $\geqq(12 s)^{3} / \mu>\mu^{2}$. Hence there is a $k$ dividing $\lambda$ for which $3 \leqq k \leqq \mu / 2 \leqq 4 s$. This $k$ must divide $c$ and so must divide $p+1$.

If $\mu=8 s$, then $\mu>8$ and so $k \geqq 4$. But from (2), $\lambda=(p+1)+3$. So $k$ must divide 3 , a contradiction. If $\mu=4 s$, (2) becomes $\lambda=2(p+1)+2$. So $k$ must divide 2 , another contradiction. Finally, if $\mu=8 s / 3$, then $k$ must be 1 which is also impossible. Hence $\mu \leqq 2 s$ and the proof is complete.

The above methods can be utilized to show that $N(g) \neq 8(g+2)$ for all $g>283$ [3].

\section{REFERENCES}

1. R. D. M. Accola, On the number of automorphisms of a closed Riemann surface, Trans. Amer. Math. Soc. 131 (1968), 398-408. MR 36 \#5333.

2. A. Hurwitz, Uber algebraishe Gebilde mit eindeutigen Transformationen in sich, Math. Ann. 41 (1893), 403-442.

3. W. T. Kiley, Automorphism groups on compact Riemann surfaces, Thesis, Brown University, Providence, R. I., 1969.

4. E. Landau, Elementare Zahlentheorie, Teubner, Liepzig, 1927; English transl., Chelsea, New York, 1958. MR 19, 1159.

5. A. M. Macbeath, Discontinuous groups and birational transformations, Proc. Summer School in Geometry and Topology, Queens College, University of St. Andrews, 1961.

6. - On a theorem of Hurwitz, Proc. Glasgow Math. Assoc. 5 (1961), 90-96. MR 26 \#4244. 
7. C. Maclachlan, A bound for the number of automorphisms of a compact Riemann surface, J. London Math. Soc. 44 (1969), 265-272. MR 38 \#4674.

8. H. Röhrl, Unbounded coverings of Riemann surfaces and extensions of rings of meromorphic functions, Trans. Amer. Math. Soc. 107 (1963), 320-346. MR 26 \#6397.

9. J. Rotman, The theory of groups, Allyn and Bacon, Boston, Mass. 1965.

10. H. Seifert and W. Threfall, Lehrbuch der Topologie, Teubner, Leipzig, 1934; reprint, Chelsea, New York, 1947.

11. H. Zassenhaus, Lehrbuch der Gruppentheorie, Teubner, Leipzig, 1937; English transl., Chelsea, New York, 1949. MR 11, 77.

UNIVERSITY OF VIRGINIA,

FAIRFAX, Virginia 22030 